\title{
An Aggregate-Inducing Peripherin Isoform Generated through Intron Retention Is Upregulated in Amyotrophic Lateral Sclerosis and Associated with Disease Pathology
}

\author{
Shangxi Xiao, ${ }^{1}$ Sonja Tjostheim, ${ }^{1}$ Teresa Sanelli, ${ }^{1}$ Jesse R. McLean, ${ }^{1}$ Patrick Horne, ${ }^{1}$ Yuxin Fan,,${ }^{2}$ John Ravits, ${ }^{2}$ \\ Michael J. Strong, ${ }^{3}$ and Janice Robertson ${ }^{1}$ \\ ${ }^{1}$ Centre for Research in Neurodegenerative Diseases, University of Toronto, Toronto, Ontario, Canada M5S 3H2, ${ }^{2}$ Neurology Section, Neurogenomics \\ Laboratory, Virginia Mason Medical Center, Benaroya Research Institute, Seattle, Washington 98101-2795, and ${ }^{3}$ Department of Clinical Neurological \\ Sciences, The University of Western Ontario, London, Ontario, Canada N6A 5A5
}

The neuronal intermediate filament protein peripherin is a component of ubiquitinated inclusions and of axonal spheroids in amyotrophic lateral sclerosis (ALS). Overexpression of peripherin causes motor neuron degeneration in transgenic mice and variations within the peripherin gene have been identified in ALS cases. We have shown previously the abnormal expression of a neurotoxic peripherin splice variant in transgenic mice expressing mutant superoxide dismutase-1. These findings indicated that abnormalities of peripherin splicing may occur in ALS. In the current study, peripherin splice variants were identified by reverse transcription-PCR of human neuronal RNA and comparisons in expression made between control and ALS spinal cord using Western blot analysis and immunocytochemistry. Using this approach we have identified a novel peripherin transcript retaining introns 3 and 4 that results in a $28 \mathrm{kDa}$ splice isoform, designated Per 28. Using an antibody specific to Per 28, we show that this isoform is expressed at low stoichiometric levels from the peripherin gene, however causes peripherin aggregation when its expression is upregulated. Importantly we show an upregulation of Per 28 expression in ALS compared with controls, at both the mRNA and protein levels, and that Per 28 is associated with disease pathology, specifically round inclusions. These findings are the first to establish that peripherin splicing abnormalities occur in ALS, generating aggregation-prone splice isoforms.

Key words: peripherin; splice variant; isoform; ALS; intron retention; intermediate filament

\section{Introduction}

Amyotrophic lateral sclerosis (ALS) is an adult-onset neurodegenerative disease affecting motor neurons of the motor cortex, brainstem, and spinal cord. $\sim 10-15 \%$ of cases are inherited [familial (fALS)] with the remainder occurring sporadically [sporadic (sALS)]. Mutations in the gene encoding superoxide dismutase-1 (SOD1) are causative of $\sim 20 \%$ of fALS cases, corresponding to $1-2 \%$ of all ALS cases (Rosen et al., 1993). Sporadic and familial ALS are clinically and pathologically indistinguishable, sharing a number of intraneuronal inclusion bodies that are characteristic of the disease (for review, see Hays, 2006). These include ubiquitinated inclusions that comprise skein-like inclusions, round inclusions and Lewy body-like inclusions (Ince et

\footnotetext{
Received July 16, 2007; revised Nov. 30, 2007; accepted Dec. 29, 2007.

J. Robertson was supported by grants from the Canadian Institutes of Health Research (CIHR), The ALS Society of Canada, The UK Motor Neurone Disease Association, The ALS Association (ALSA), and The Muscular Dystrophy Association (MDA), and holds a Canada Research Chair in ALS. S.X. was supported by a CIHR Postdoctoral Training Fellowship. J. Ravits was supported by National Institutes of Health Grant R21.NS051738, the Benaroya Foundation, and the Juniper Foundation. M.J.S. was supported by ALSCanada, the Scottish Rite Charitable Foundation, ALSA, and the MDA.

Correspondence should be addressed to Dr. Janice Robertson, Centre for Research in Neurodegenerative Diseases, University of Toronto, Tanz Building, 6 Queen's Park Crescent West, Toronto, Ontario, Canada M5S 3H2. E-mail:jan.robertson@utoronto.ca.

DOI:10.1523/JNEUROSCI.3222-07.2008

Copyright $\odot 2008$ Society for Neuroscience $\quad$ 0270-6474/08/281833-08\$15.00/0
}

al., 1998a; Hays, 2006). Hyaline conglomerate inclusions are also ubiquitinated, but appear to be more closely linked with fALS cases carrying mutations in SOD1 (Ince et al., 1998b; Hays et al., 2006). Peripherin, a neuronal intermediate filament protein, is associated with ubiquitinated inclusions, specifically round inclusions and Lewy body-like inclusions, hyaline conglomerate inclusions, as well as with axonal spheroids, large swellings that occur in proximal axons of diseased motor neurons (Corbo and Hays, 1992; Migheli et al., 1993; He and Hays, 2004; Xiao et al., 2006). Together with the association of peripherin with intraneuronal inclusions, there is also a generalized increased in peripherin immunoreactivity in both the perikarya and axons of motor neurons affected by the disease (Robertson et al., 2002; Robertson et al., 2003; Xiao et al., 2006). Overexpression of peripherin induces motor neuron degeneration in transgenic mice and variations within the peripherin gene have been identified in a few ALS cases. This combined evidence supports a role for peripherin in the pathogenesis of ALS.

We have shown previously the deregulated expression of peripherin splice variants in transgenic mouse models of ALS (Robertson et al., 2003). In particular, we showed the abnormal expression of a neurotoxic splice variant of peripherin, Per 61, in motor neurons of mutant SOD1 transgenic mice (Robertson et al., 2003). This splice variant was generated by the retention of 
intron 4 and its upregulated expression induced peripherin aggregate formation and motor neuronal death (Landon et al., 1989, 2000; Robertson et al., 2003). These findings indicated that abnormalities of peripherin splicing may be relevant to the disease mechanisms in ALS.

In this regard, we set out to find the human equivalent of Per 61. However, in mouse, intron 4 is $96 \mathrm{bp}$ in length, whereas in human it is $91 \mathrm{bp}$. As such, the complete retention of intron 4 in human would lead to a frameshift and generation of a C-terminally truncated protein of $32 \mathrm{kDa}$. Therefore the splicing event that generates Per 61 in mouse cannot occur in human. Nevertheless, the existence of a human peripherin expressed sequence tag (EST) sequence retaining part of intron 4 indicated that read through into this intron could occur. In our attempt to amplify transcripts retaining intron 4 , we inadvertently identified a transcript retaining introns 3 and 4 . This transcript encodes a C-terminally truncated protein of $28 \mathrm{kDa}$ derived from translation of the $5^{\prime}$ end of peripherin up to a stop codon 30 bp into intron 3. This peripherin splice variant, designated Per 28, is the first to be identified in human and is completely distinct from Per 61 in mouse. We have shown that there is upregulated expression of Per 28 at both the mRNA and protein levels in ALS, and that this may be associated with inclusion body formation. Collectively our findings establish that splicing abnormalities of peripherin occur in ALS generating an aggregation-prone splice variant, Per 28.

\section{Materials and Methods}

RNA and semiquantitative RT-PCR. Human RNA samples for reverse transcription (RT)-PCR were obtained commercially either from Clontech (Palo Alto, CA) or Ambion (Austin, TX) and treated with DNase before RT-PCR to remove any genomic DNA contamination of the RNA sample. Control RT-PCR experiments with omission of the reverse transcriptase were performed in parallel with all experiments to further ensure results obtained were not caused by genomic contamination. Premium total RNA from human dorsal root ganglia was from Clontech (catalog number CR2496). Control total RNA samples from human lumbar spinal cord was pooled from 49 male/female Caucasians age 15 to 66 (catalog number 64113-1; Clontech), and from a normal 65-year-old male Caucasian (catalog number B6840; Ambion). Total RNA from ALS human lumbar spinal cord (catalog number B6162; Ambion) was from two 70-year-old male Caucasians. The cDNAs were synthesized from 1 $\mu \mathrm{g}$ of total RNA with oligo-(dT) ${ }_{20}$ using the SuperScript III First-Strand Synthesis System for RT-PCR from Invitrogen (Burlington, Ontario, Canada) following the manufacturer's protocol. The ratio of mRNA transcripts was estimated using semiquantitative RT-PCR normalized using primers specific for $\beta$-actin (Ambion) and choline acetyltransferase (ChAT) (for primer sequences, see Table 1). For amplifying the normal peripherin gene transcript $P R P H$, primers were located in exons 3 and 6 (Table 1). For PCR, $1 \mu \mathrm{l}$ of template cDNA solution was placed in $25 \mu \mathrm{l}$ of reaction solution containing $1 \mathrm{x}$ PCR buffer $(20 \mathrm{~mm}$ Tris- $\mathrm{HCl}, \mathrm{pH}$ 8.4, $50 \mathrm{~mm} \mathrm{KCl}$ ), $200 \mu \mathrm{M}$ dNTP, $2 \mathrm{~mm} \mathrm{MgCl}_{2}, 5 \%$ DMSO, $2 \mu \mathrm{M}$ each primer, and 2 U Platinum TaqDNA Polymerase (Invitrogen). The amplification conditions for the peripherin splice variant consisted of initial denaturation at $95^{\circ} \mathrm{C}$ for $5 \mathrm{~min}$, followed by 40 cycles of $95^{\circ} \mathrm{C}$ for $30 \mathrm{~s}$, $62^{\circ} \mathrm{C}$ for $30 \mathrm{~s}$, and $72^{\circ} \mathrm{C}$ for $30 \mathrm{~s}$ in a GeneAmp PCR system 9700 (Applied Biosystems, Streetsville, Ontario, Canada). Cycle numbers for the RTPCR amplifications of $\beta$-actin, ChAT, and $P R P H$ were 25,35 , and 30 cycles, respectively.

Cloning of full-length Per 3,4 cDNA using 5' and 3' RACE. According to the sequence of intron 4 of peripherin obtained from the University of California at Santa Cruz (UCSC) Genome Browser (accession number,
NM_006262), we designed 5' and $3^{\prime}$ gene-specific primers (GSPs): an antisense primer (GSP1) for $5^{\prime}$-rapid amplification of cDNA ends (RACE) PCR (5'-GAC AGG TCC GCG TAC TGA GAA GTG G-3') and a sense primer (GSP2) for $3^{\prime}$-RACE PCR ( $5^{\prime}$-GTC CAA GGT GCA AGA GCC GGG AGG-3'). 5' -RACE-ready cDNA was synthesized by combining $1 \mu \mathrm{g}$ of total RNA with BD SMART II A Oligonucleotide (BD Biosciences, Mississauga, Ontario, Canada) and 5'-RACE coding sequence (CDS) primer; 3 '-RACE-ready cDNA was synthesized by combining 1 $\mu \mathrm{g}$ of total RNA with $3^{\prime}$-CDS primer. CDS primers were supplied by the manufacturer. The detailed procedure is exactly as described in the BD SMARTTM RACE cDNA Amplification Kit user manual (BD Biosciences; catalog number 634914). Fifty microliters of RACE PCR solution consisted of $2.5 \mu \mathrm{l}$ of $5^{\prime}$-RACE-ready cDNA or 3'-RACE-ready cDNA, $34.5 \mu \mathrm{l}$ of PCR-grade water, $5 \mu \mathrm{l}$ of 10x Advantage 2 PCR buffer, $1 \mu \mathrm{l}$ of $10 \mathrm{~mm}$ dNTP, $1 \mu \mathrm{l}$ of 50x BD Advantage 2 Polymerase Mix, $5 \mu \mathrm{l}$ of 10x Universal Primer Mix, and $1 \mu \mathrm{l}$ of $10 \mu \mathrm{M}$ GSP1 or GSP2. Thermal cycling was followed using the touchdown PCR program: five cycles at $94^{\circ} \mathrm{C}$ for $30 \mathrm{~s}, 72^{\circ} \mathrm{C}$ for $3 \mathrm{~min}$; five cycles at $94^{\circ} \mathrm{C}$ for $30 \mathrm{~s}, 70^{\circ} \mathrm{C}$ for $30 \mathrm{~s}$, $72^{\circ} \mathrm{C}$ for $3 \mathrm{~min}$; and 30 cycles at $94^{\circ} \mathrm{C}$ for $30 \mathrm{~s}, 68^{\circ} \mathrm{C}$ for $30 \mathrm{~s}, 72^{\circ} \mathrm{C}$ for 3 $\mathrm{min}$. The $5^{\prime}$-RACE and $3^{\prime}$-RACE PCR products were analyzed by electrophoresis on $1.2 \%(\mathrm{w} / \mathrm{v})$ agarose/ethidium bromide gels and purified using the MiniElute Gel Extraction kit (catalog number 28604; Qiagen, Mississauga, Ontario, Canada). The isolated fragments were cloned directly into a topoisomerase (TOPO) cloning vector: pCR2.1-TOPO (catalog number K4510-20; Invitrogen). The inserts were sequenced with M13 forward and reverse primers on an ABI PRISM 3100 Genetic Analyzer (Applied Biosystems). The full-length cDNAs were generated by blunt-end ligation of the $5^{\prime}$ fragment and 3 ' fragment, then subcloned into the BamH1/EcoRI sites of pcDNA3.1 (catalog number V795-20; Invitrogen) and subjected to sequence analysis for final verification.

Cloning of full-length human peripherin gene $\mathrm{PRPH}$. A probe specific to the human peripherin gene was used to identify bacterial artificial chromosome (BAC) clone number 977B10 from the RPCI-11 Human BAC Clones available from the Canadian Institutes of Health Research Genome Resource Facility. The BAC DNA was extracted from a bacterial culture inoculate using alkaline lysis and the full-length human peripherin gene was obtained from $977 \mathrm{~B} 10$ BAC DNA by simultaneous digestion with EcoRI and EcoRV. A band corresponding to $\sim 5000$ bp was purified using the MiniElute Gel Extraction Kit (Qiagen), subcloned into the EcorR1/EcoRV site of pcDNA3.1(-), and subjected to sequence analysis for verification.

Transient transfection. A human adrenal carcinoma cell line, SW13 vimentin $(-)$ [SW13vim $(-)$ ], was transfected using Lipofectamine 2000 (Invitrogen) following manufacturer instructions. Ectopic expression of peripherin was detected using peripherin polyclonal antibody (AB1530; Millipore, Temecula, CA).

Preparation of siRNA targeting Per 3,4. The selected small interfering RNA (siRNA) sequences for Per 3,4 was from nucleotides 129-137 for RNAi-1 and 262-280 for RNAi-2, relative to the start of intron 3. The sequences were designed using an siRNA design program (Dharmacon, Lafayette, CO) and a BLAST search revealed no substantial homology of the chosen sequences to other genes. The oligonucleotides were synthesized by Integrated DNA Technologies (Coralville, IA) and diluted to 1 $\mu \mathrm{g} / \mu \mathrm{l}$. The forward and reverse primers were annealed and cloned into linearized pSupressorNeo vector according to the manufacturer's protocol (Imgenex, San Diego, CA). 
Immunocytochemistry of cultured cells. SW13vim(-) cells grown on glass coverslips were fixed in methanol for $5 \mathrm{~min}$ at $-20^{\circ} \mathrm{C}$ and rehydrated in PBS. Immunocytochemistry was performed using an antibody recognizing peripherin (AB1530) and used at 1:1000 diluted in PBS. Antibody distribution was visualized by epifluorescence microscopy after incubation with secondary antibodies, with Alexa Fluor 594 diluted 1:350 in PBS (Invitrogen).

Immunoblotting. Cells were harvested in $62.5 \mathrm{~mm}$ Tris, $\mathrm{pH}$ 6.8, containing $2 \%$ SDS and $10 \%$ glycerol, and assayed for total protein using the bicinchoninic acid assay (Sigma, St. Louis, MO). Loadings of 10-100 $\mu \mathrm{g}$ of protein were analyzed on $10 \%(\mathrm{w} / \mathrm{v})$ SDS-polyacrylamide gels and then blotted to polyvinyldiflouride membrane. For immunoblotting, membranes were incubated with antibodies recognizing peripherin (MAB1527 or AB1530) diluted 1:5,000 in blocking solution (3\% skim milk powder in TBS-Tween). To detect the Per 28 splice variant, a rabbit polyclonal antiserum was raised to a synthetic peptide corresponding to the sequence derived from translation of the first $30 \mathrm{bp}$ of intron 3 (VSGPGIRGGF) and used at a dilution of 1:3000. Antibody binding was revealed with the ECL detection system (PerkinElmer, Waltham, MA).

Primary motor neuron cultures. The same protocol was used as described previously for our studies on Per 61 (Robertson et al., 2003). In brief, dissociated spinal cord cultures were prepared as described previously (Durham et al., 1997; Robertson et al., 2001, 2003). The Per 3,4 cDNA in pcDNA 3.1 at $100 \mathrm{ng} / \mathrm{ul}$ was microinjected into motor neuron nuclei along with the fluorescent marker dextran-FITC $(15 \mathrm{mg} / \mathrm{ml}$; Invitrogen). Viability was assessed daily by counting the number of motor neurons containing the marker. The number of viable motor neurons counted on each day was normalized to the number present on day 1 after microinjection. Experiments were performed in triplicate. For immunocytochemistry, cultures were fixed in 4\% paraformaldehyde in PBS for 15 min, and then in blocking solution [as for the SW13vim(-) cells] for 30 min. Double labeling was performed using the Per 28 antibody (diluted $1: 1000)$ and monoclonal peripherin antibody (MAB1527, diluted 1:1000) followed by secondary antibody detection, Alexa Fluor 594 or 488 , respectively.

Human spinal cord tissue. Triton X-100 (TX-100) extractions were prepared from $\sim 100 \mathrm{mg}$ of lumbar spinal cord tissue taken from four sporadic ALS cases and four controls. The control cases comprised one with no indication of neurological disease and three with neurological disease (two multisystems atrophy and one corticobasal degeneration). Briefly, samples were homogenized in $1 \mathrm{ml}$ high salt buffer (HSB; $50 \mathrm{~mm}$ Tris, pH7.5, $750 \mathrm{~mm} \mathrm{NaCl}, 5 \mathrm{~mm}$ EDTA) containing protease inhibitor mixture (Sigma) and centrifuged at high speed in an Eppendorf (Mississauga, Ontario, Canada) microcentrifuge at $4^{\circ} \mathrm{C}$ for $10 \mathrm{~min}$. The resulting pellet was rehomogenized in HSB containing 1\% TX-100 and centrifuged as before. This extraction was repeated twice. The final pellet was then homogenized in HSB containing $1 \mathrm{M}$ sucrose and centrifuged as before to remove myelin. Equal protein amounts from the different samples were resolved by $10 \%$ SDS-PAGE and probed by immunoblotting with Per 28-specific antisera or polyclonal peripherin antibody (AB1530). For immunocytochemistry, paraffin embedded sections (6 $\mu \mathrm{m})$ of lumbar spinal cord were rehydrated through a series of washes in graded ethanol and finally in water. Sections were pretreated with $10 \mathrm{~mm}$ sodium citrate, $\mathrm{pH}$ 6.0, for epitope retrieval and incubated with Per 28 splice variant-specific antisera diluted 1:1000 in DakoCytomation (Mississauga, Ontario, Canada) Antibody Diluent overnight at $4^{\circ} \mathrm{C}$. Antibody labeling was revealed using the DakoCytomation EnvisonTM System according to the manufacturer's instructions using 3,3'-diaminobenzidine as chromagen. Labeled sections were visualized using a Leica (Richmond Hill, Ontario, Canada) DM 6000 microscope and digital images obtained with a MicroPublisher 3.3 RTV color camera and Openlab imaging software (Improvision, Lexington, MA).

\section{Results}

Identification of a peripherin transcript retaining introns 3 and 4

We identified two EST sequences corresponding to splice variants of human peripherin that retain part of intron 4 or intron 3 (BE786797 and BI832203, respectively) using the UCSC Genome

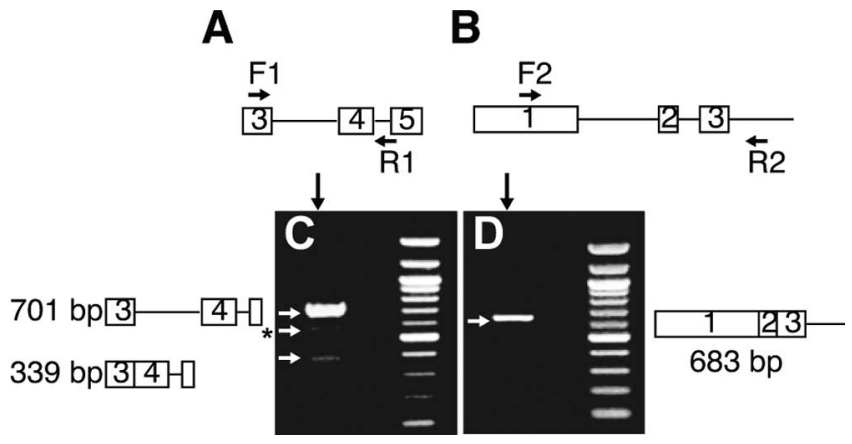

Figure 1. Identification of a peripherin transcript retaining introns 3 and 4 . A, Schematic showing locations of forward primer $\mathrm{F} 1$ in exon 3 and reverse primer $\mathrm{R} 1$ in intron 4/exon 5 used for RT-PCR amplification of transcripts retaining intron 4. $\boldsymbol{B}$, Off-target primer locations $F 1$ in exon 1 and R2 in intron 3 used to control for genomic contamination. C, A major RT-PCR product of 701 bp was amplified from human dorsal root ganglion RNA using the primer pair indicated in $A$ corresponding to a transcript retaining introns 3 and 4; a minor transcript of 339 bp encompassing intron 4 was also identified, as well as product of $550 \mathrm{bp}$, which could not be identified. $\boldsymbol{D}$, The primer pair in $\boldsymbol{B}$ amplified a product of $683 \mathrm{bp}$ corresponding to a transcript in which introns 1 and 2 were spliced out, providing additional confirmation that the samples were not contaminated with genomic DNA. All RT-PCR products were confirmed by sequencing.

Browser. Primers for RT-PCR were designed according to the retained sequence of intron 4 because we had found that peripherin splicing events involving retention of intron 4 occurred in mutant SOD1 transgenic mice (Robertson et al., 2003). Premium total RNA extracted from human dorsal root ganglia (Clontech) was chosen as the source for RT-PCR to identify peripherin splice variants because peripherin is highly expressed in this tissue (Wong and Oblinger, 1990). Because our aim was to identify splicing events involving intron retention, a number of steps were taken to avoid possible confounding results caused by genomic contamination. First, all of the RNA samples were treated with DNase before RT-PCR. Second, control experiments were performed in which reverse transcriptase was omitted showing that there was no PCR amplification of genomic DNA. Third, the first strand synthesis was performed using oligo-(dT)20 to amplify mRNA only. Fourth, parallel RT-PCR experiments were performed using off-target primers located in exon 1 and intron 3 (Fig. $1 B$ ). The product generated from these reactions had introns 1 and 2 spliced out (Fig. $1 D$, arrow), confirming that our samples were not contaminated with genomic DNA. Using a forward primer located in exon 3 of peripherin and a reverse primer in intron 4 (Fig. $1 A$, Table 1), one major product of $701 \mathrm{bp}$ was generated by RT-PCR, which was shown by sequencing to correspond to a transcript retaining introns 3 and 4 (Fig. 1C). Two minor products were also detected, one of $339 \mathrm{bp}$ corresponded to a transcript retaining intron 4 only, the other (Fig. $1 C$, asterisk) could not be identified. These transcripts were in low abundance as only the constitutively spliced peripherin isoform (in which all introns are spliced out) was detected when RT-PCR was performed using primers located in the $5^{\prime}$ and $3^{\prime}$ untranslated regions (data not shown). Therefore $5^{\prime}$ and $3^{\prime}$ RACE using genespecific primers in intron 4 (supplemental Fig. 1, available at www.jneurosci.org as supplemental material) was used to obtain the full-length transcript retaining introns 3 and 4, which was called Per 3,4 cDNA.

\section{Expression of Per 3,4 in transfected cells}

Expression of the Per 3,4 cDNA was compared with the normal full-length human peripherin gene in transfected SW13vim(-) cells, a human cell line lacking an endogenous intermediate fila- 
ment network (Sarria et al., 1990). The constitutive expression product from the peripherin gene, in which all intronic sequences are spliced out, has a molecular weight of $\sim 58 \mathrm{kDa}$ (Per 58) according to SDS-PAGE (Fig. 2A). For Per 3,4, the retention of intron 3 introduces a premature stop codon $30 \mathrm{bp}$ downstream from the start of the intron, predicting a peripherin species of $28 \mathrm{kDa}$ with a unique 10 amino acid sequence at the C-terminus. Immunoblots of cell lysates from transfected cells probed with commercially available peripherin antibody (AB1530; Millipore) showed the expected expression of Per 58 from the peripherin gene (Fig. $2 \mathrm{~A}$, lane 1). In lysates from cells expressing the Per 3,4 cDNA, a species of $\sim 28 \mathrm{kDa}$ was apparent, which corresponded to the molecular weight predicted from the $\mathrm{CDNA}$ sequence and was designated Per 28 (Fig. 2A, lane 2, large arrow). The identity of this species was confirmed using a specific antibody raised to the unique 10 amino acid sequence derived from translation of the initial 30 bp of intron 3 (Fig. 2 B). There was also expression of Per 58 from the Per 3,4 cDNA, as well as expression of four other minor species of $\sim 48$, $\sim 45$, $\sim 32$, and $\sim 25 \mathrm{kDa}$ (Fig. $2 \mathrm{~A}$, lane 2 , small arrows). Expression of Per 58 from the Per 3,4 cDNA indicates that the Per 3,4 transcript undergoes splicing to remove introns 3 and 4 in transfected SW13vim $(-)$ cells. The processing events that generated the four additional peripherin species are unknown, but unlikely to involve post-translational modifications because they are absent in similar loadings of lysates from cells expressing the peripherin gene (Fig. $2 \mathrm{~A}$, compare lanes 1,2).

As protein loadings were increased, we found that all of the species expressed from Per 3,4 cDNA were also expressed at low levels from the peripherin gene (Fig. $2 A$, lane 7 , arrows). This included Per 28, verified using the specific antisera (Fig. $2 \mathrm{~B}$, lane 1). However, Per 28 was expressed at a relatively higher level from the Per 3,4 cDNA than from the wild-type peripherin gene. These findings indicated that the additional peripherin species are in fact normal expression products from the peripherin gene, but are expressed at lower levels and at different relative stoichiometric levels compared with expression from the Per 3,4 cDNA.

Changes in peripherin isoform expression causes aggregation In SW13vim $(-)$ cells expressing the human peripherin gene peripherin displayed the normal filamentous networks that we described previously for the mouse gene (Fig. 3A) (Beaulieu et al., $1999 b)$. In contrast, expression of the Per 3,4 cDNA led to the generation of large aggregates (Fig. $3 B$, arrows). As the same peripherin species are expressed from the peripherin gene as from the Per 3,4 cDNA, this indicated that a change in the relative ratios of peripherin isoform expression induces peripherin aggregate formation. To verify this, we designed an interfering RNA targeting intron 3, which when coexpressed with the Per 3,4 cDNA would lead to a downregulation of Per 28. Of two siRNAs designed, siRNA2, but not siRNA1, downregulated expression of transcripts retaining intron 3 (Fig. 4A, compare lanes 2, 3) and correspondingly reduced expression of Per 28 while maintaining expression of Per 58 (Fig. 4 B, compare lanes 1, 2, Per 28 indicated

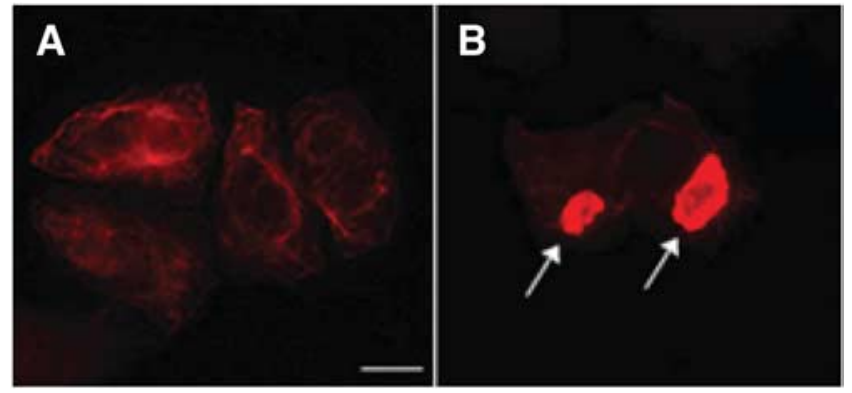

Figure 3. $\quad \boldsymbol{A}, \boldsymbol{B}$, Immunofluorescence labeling of SW13vim $(-)$ cells transfected with the normal peripherin gene $(\boldsymbol{A})$ compared with the Per 3,4 CDNA $(\boldsymbol{B})$, labeled with polyclonal peripherin antibody (AB1530). Note the normal filamentous distribution of peripherin in $A$ and the large amorphous aggregates (indicated by arrows) in $\boldsymbol{B}$. Scale bar, $10 \mu \mathrm{m}$.

with large arrow). This change in the relative expression levels of peripherin isoforms caused Per 3,4 to form filaments instead of aggregates (Fig. 4, compare $C, D$ ), indicating that alterations in the relative expression levels of peripherin isoforms determines whether peripherin forms filaments or aggregates.

\section{Expression of Per 3,4 forms inclusion bodies in primary motor neurons}

We had shown previously that expression of the mouse splice variant Per 61 formed punctate aggregates and was neurotoxic to motor neurons in culture (Robertson et al., 2003). We therefore tested the effects of Per 3,4 expression on motor neuron viability using intranuclear microinjection of the Per 3,4 plasmid or plasmid alone, as described previously (Fig. 5A) (Robertson et al., 2003). Expression of Per 3,4 in motor neurons led to the formation of singular inclusion bodies in the perikarya, very different from the punctate aggregates we previously observed for Per 61 (Fig. 5B) (Robertson et al., 2003), but more reminiscent of peripherin pathology observed in ALS (Xiao et al., 2006). The viability assay indicated that although Per 3,4 was associated with 

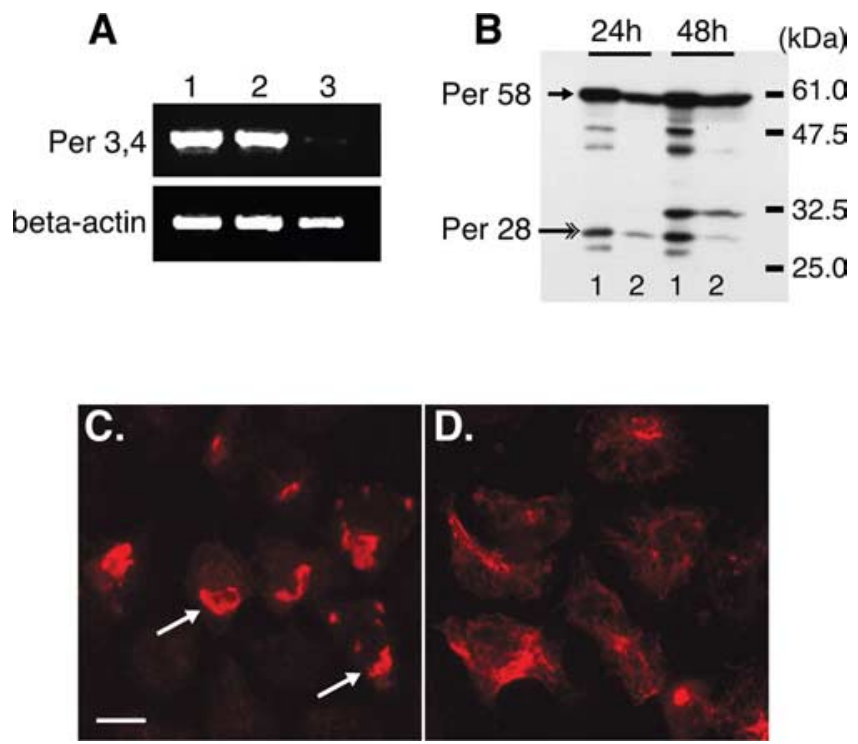

Figure 4. Downregulation of Per 28 using RNAi prevents aggregate formation. A, RT-PCR of RNA isolated from SW13vim( - ) cells transfected with Per 3,4 (lane 1); Per 3,4 and RNAi-1 (lane 2); and Per 3,4 and RNAi-2 (lane 3). Samples were normalized to $\beta$-actin. Note the downregulation of Per 3,4 message in cells cotransfected with RNAi-2 (lane 3 ). $\boldsymbol{B}$, Immunoblot of total cell lysates of cells transfected with Per 3,4 (lanes 1) or cotransfected with Per 3,4 and RNAi-2 (lane 2), probed with AB1530. Note the reduction in expression of Per 28 at 24 and $48 \mathrm{~h}$ after expression (lane 2, large arrow) and the maintained expression of the normal peripherin product (lanes 1, 2, small arrow). C, D, Immunofluorescence labeling of SW13vim(-) cells expressing Per 3,4 (C) or Per 3,4 and RNAi-2 (D), labeled with AB1530. Note the peripherin aggregates in C and the predominance of filamentous networks in $\boldsymbol{D}$. Scale bar, $30 \mu \mathrm{m}$.

some neurotoxicity, the effect was not as dramatic as that observed previously for Per 61 (Fig. 5A) (Robertson et al., 2003).

\section{Per 3,4 transcript expression is upregulated in ALS}

Because upregulated expression of Per 28 induced peripherin to aggregate and form structures resembling peripherinimmunoreactive inclusion bodies in ALS, we investigated whether there was an upregulation of Per 3,4 transcript expression in ALS spinal cord tissue compared with controls using semiquantitative RT-PCR. Because the cellular profile is different in disease spinal cord tissue compared with normal tissue (loss of motor neurons and proliferation of nonneuronal inflammatory cells, i.e., microglia and astrocytes in disease tissue), two sets of primers were used for normalization: $\beta$-actin to normalize for total mRNA, and ChAT to normalize for total neuronal mRNA. Findings showed a dramatic upregulation of the Per 3,4 transcript in ALS spinal cord compared with controls whether $\beta$-actin or ChAT was used for normalization (Fig. 6, compare lanes 1, 2 with 3, 4). The normal peripherin transcript $(P R P H)$ was also upregulated when samples were normalized to ChAT, consistent with our previous findings showing an upregulation of peripherin expression in ALS (Robertson et al., 2003).

\section{Per 28 expression is upregulated in ALS}

We next tested whether the increase in expression of the Per 3,4 transcript in ALS tissue led to a corresponding increased expression of Per 28. Equivalent amounts of TX-100 preparations from lumbar spinal cord of four ALS cases and four control cases were probed by immunoblotting using the Per 28-specific antisera (Fig. $7 \mathrm{~A}$ ). The control samples were comprised of one non-neurological disease case (lane 8 ) and three with neurological diseases other than ALS (two multisystem atrophy, lanes 5 and 6 , and one corticobasal degeneration, lane 7). The details of the cases used for the study are shown in supplemental Table 1 (available at www.jneurosci.org as supplemental material). The results in Figure $7 \mathrm{~A}$ show that there is a pronounced increase in expression of Per 28 in all four of the ALS cases with expression below detectable limits in the control cases. Reprobing of the blots with commercially available peripherin antibody $(\mathrm{AB} 1530)$ showed the increase in Per 58 expression in the ALS cases relative to controls that we have reported previously (Fig. 7 B) (Robertson et al., 2003). However, this generalized increase in peripherin expression was not sufficient to account for the substantive increase in signal detected for Per 28 in the ALS samples.

\section{Association of Per 28 with peripherin pathology in ALS}

We have shown that there is an upregulation of the Per 3,4 splicing intermediate in ALS spinal cord compared with controls, and a corresponding increased expression of Per 28, the major expression product from the Per 3,4 transcript. Because we have shown that upregulated expression of Per 3,4 induces peripherin aggregate formation, we investigated whether Per 28 was associated with peripherin pathology in ALS. Immunocytochemical labeling of ALS spinal cord tissue with the Per 28 antibody showed distinct labeling of peripherin aggregates resembling round inclusions in the cytoplasm of motor neurons (Fig. 8). There was no labeling of control spinal cord. These findings indicated that not only was there increased expression of Per 28 in ALS, but that it was also associated with disease pathology.

\section{Discussion}

Peripherin is a type III intermediate filament protein associated with the major disease pathologies in ALS including ubiquitinated round inclusions and Lewy body-like inclusions (He and Hays, 2004; Xiao et al., 2006). Overexpression of peripherin in transgenic mice induces motor neuron degeneration that is accompanied by the presence of intraneuronal aggregates of peripherin (Beaulieu et al., 1999a). We have shown previously the expression of a neurotoxic splice variant of peripherin in motor neurons of transgenic mice expressing mutant SOD1 (Robertson et al., 2003). Although overexpression or ablation of peripherin in these mice did not affect disease course (Lariviere et al., 2003), it is now known that motor neuron degeneration caused by mutant SOD1 in transgenic mice is multifactorial, involving both neuronal and nonneuronal components (Clement et al., 2003) and discussed in (Xiao et al., 2006). Furthermore, mutations in SOD1 are causative of only $1-2 \%$ of all fALS, whereas peripherin abnormalities are found broadly in both fALS and sALS (Corbo and Hays, 1992; Migheli et al., 1993; He and Hays, 2004). The neurotoxic splice variant we described previously in mouse, Per 61, was generated through the in-frame retention of intron 4, which is 96 bp in length and introduces a 32 amino acid insertion within a highly conserved domain of the peripherin protein (Landon et al., 1989, 2000; Robertson et al., 2003). Although a Per 61 antibody labeled diseased motor neurons in ALS and immunoprecipitated a higher molecular weight peripherin species, the transcript underlying the generation of this species remains to be identified (Robertson et al., 2003). Indeed the splicing event that generates Per 61 in mouse cannot occur in human, as intron 4 in human is $91 \mathrm{bp}$ in length and its complete retention would introduce a premature stop 
codon and the generation of a truncated peripherin species of $\sim 32 \mathrm{kDa}$. Nevertheless, evidence from EST sequences indicated that read through into intron 4 could occur in human. As such, it was through our attempts to identify human peripherin transcripts retaining intron 4 that we identified the Per 3,4 transcript, which retains both introns 3 and 4 . Because this transcript was derived from intron retention, we were particularly cautious of spurious results obtained from possible genomic contamination. To confirm that this transcript was not caused by genomic contamination all of our RNA samples were pretreated with DNase before RT-PCR, controls omitting reverse transcriptase showed no amplification of genomic DNA, oligo$(\mathrm{dT})_{20}$ was used for first strand synthesis and the use of off-target primers showed

the proper splicing of introns 1 and 2. This transcript was in low relative abundance compared with the constitutively spliced transcript, encoding Per 58, and could only be amplified using gene-specific primers within either introns 3 or 4 . Intron retention is the least frequent of alternative splicing events in mammals, and as such, is the least studied (Clark and Thanaraj, 2002; Kan et al., 2002). The retention of intron 3 leads to the introduction of a premature stop codon $30 \mathrm{bp}$ downstream from the start of the intron, generating a truncated protein of $\sim 28 \mathrm{kDa}$ with a unique 10 amino acid sequence at the C-terminus, designated Per 28. Using a specific antisera generated to the unique 10 amino acid sequence, we have established the existence of Per 28 as an alternatively spliced isoform expressed from the Per 3,4-cDNA and at low levels from the wild-type human peripherin gene. Additional minor peripherin species were also identified of $\sim 25, \sim 32$, $\sim 45$, and $\sim 48 \mathrm{kDa}$ expressed from the Per 3,4-cDNA and at substoichiometric levels from the peripherin gene. We have positively identified the $45 \mathrm{kDa}$ species as being derived from the use of an downstream, in-frame alternative initiation codon within the peripherin transcript (McLean et al., 2008) and we are currently investigating the processing events leading to the generation of the other species. Remarkably, the deregulated expression of peripherin isoforms from the Per 3,4-cDNA induced peripherin to form aggregates instead of filaments, raising the possibility that deregulated peripherin splice isoform expression may contribute to peripherin aggregation and inclusion body formation in ALS. This premise was supported by experiments in which the peripherin splicing profile of Per 3,4 was reverted using RNAi to downregulate the expression of Per 28 relative to Per 58, consequently inducing peripherin to form filaments instead of aggregates. We have provided direct evidence that there is a similar deregulation of peripherin splicing in ALS by showing an upregulation of the Per 3,4 mRNA relative to the constitutively spliced peripherin transcript in ALS lumbar spinal cord compared with controls, and that this corresponded with upregulated expression of Per 28 . We also showed the association of Per 28 with round inclusion bodies in affected motor neurons, providing additional support for the relevance of abnormalities in peripherin splicing contributing to the pathogenic mechanism(s) underlying ALS by generating aggregation-prone isoforms.

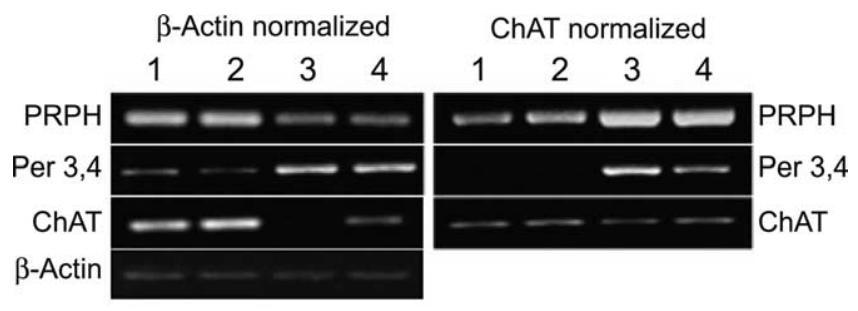

Figure 6. Upregulated expression of Per 3,4 mRNA in ALS versus control tissue. RT-PCR of total RNA isolated from spinal cords of control (lanes 1, 2) and ALS (lanes 3, 4) cases. Samples were normalized to $\beta$-actin or to ChAT (to normalize for neuronal content). Note the reduction in ChAT RT-PCR product in ALS samples (lanes 3, 4) compared with controls (lanes 1, 2) in the reactions normalized to $\beta$-actin, consistent with loss of motor neurons in ALS. The normal peripherin message was amplified using the primers shown in Table 1 (PRPH). PRPH appeared decreased in ALS samples (lanes 3, 4) compared with controls (lanes 1, 2) when RT-PCRs were normalized to $\beta$-actin, but increased when normalized to ChAT. An increase in Per 3,4 expression was detected in ALS samples compared with controls whether the samples were normalized to $\beta$-actin or to ChAT.

To test whether the inclusions formed by Per 28 were neurotoxic, we expressed the Per 3,4 cDNA in primary motor neurons by intranuclear microinjection. Although Per 28 expression formed inclusions in the perikarya of motor neurons, the presence of these inclusions was only associated with mild neurotoxicity. This is distinct from our earlier findings on the mouse splice variant Per 61, which was extremely neurotoxic when expressed in motor neurons (Robertson et al., 2003). However, Per 28 is very different from Per 61. Per 28 encodes the $\mathrm{N}$-terminal region of peripherin up to the distal end of coil $1 \mathrm{~b}$ of the $\alpha$ helical rod domain and has a unique amino sequence, VSGPGIRGGF, at the C-terminus. In contrast, Per 61 incorporates a 32 amino acid insertion within coil 2 of the rod domain, but otherwise has the same sequence as Per 58 (Landon et al., 1989, 2000). Expression of Per 28 in transfected SW13 cells or primary motor neurons was associated with the formation of singular inclusion bodies, whereas Per 61 formed punctate aggregates, dispersed throughout the cytoplasm (Robertson et al., 2003). Therefore based on structural considerations alone, it would be anticipated that the biological effects of Per 61 compared with Per 28 would be entirely different.

Here, we have identified a novel human peripherin splice variant, Per 28 , that is encoded by a transcript retaining in- 
A

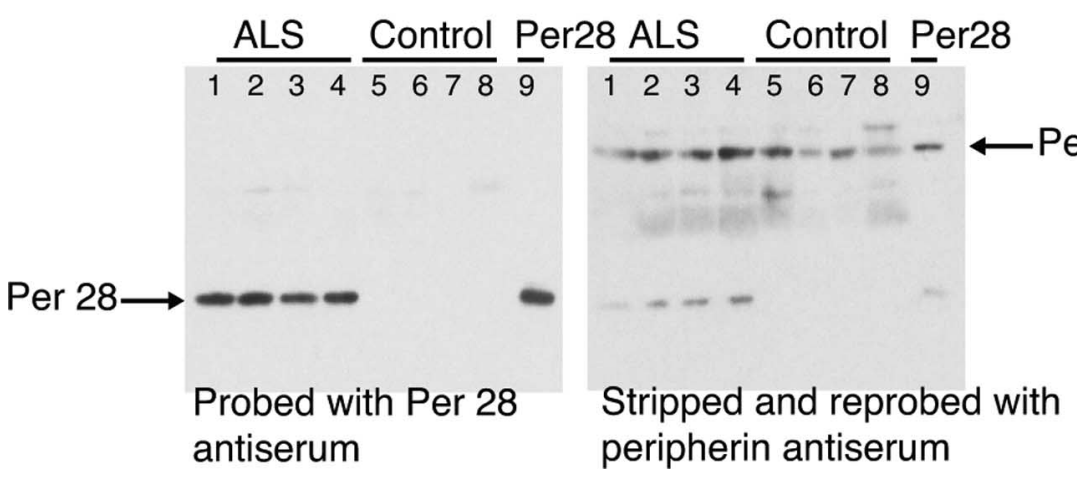

Figure 7. Upregulated expression of Per 28 in ALS versus control tissue. A, Triton X-100 (30 $\mu \mathrm{g})$ preparations from lumbar spinal cord of four independent ALS and control cases were probed by immunoblotting with the Per $28 \mathrm{kDa}$ splice variant-specific antiserum. Per 28 was detected in the ALS samples but not in the controls. $\boldsymbol{B}$, The immunoblot in $\boldsymbol{A}$ was stripped and reprobed with $A B 1530$, the commercially available antiserum, revealing the constitutively spliced Per 58 kDa peripherin species in all samples.
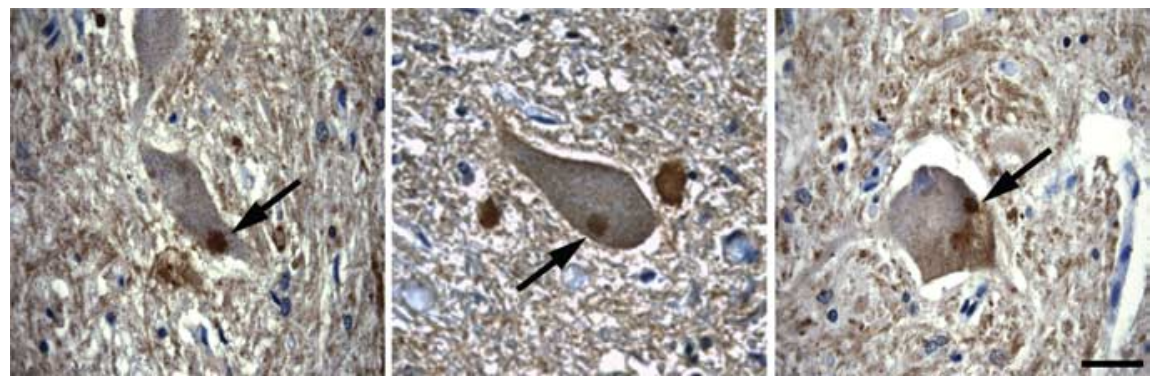

Figure 8. Immunocytochemical labeling of ALS lumbar spinal cord with Per 28-specific antiserum. $\boldsymbol{A}-\boldsymbol{C}$, Three examples of motor neurons containing Per 28 immunoreactive inclusion bodies (arrows). Scale bar, $20 \mu \mathrm{m}$.

trons 3 and 4. Upregulated expression of Per 28 induces formation of peripherin inclusions, both in transfected SW13vim(-) cells and in primary motor neurons. Importantly we have shown using a splice variant-specific antibody that Per 28 expression is upregulated in ALS spinal cord, and that Per 28 is associated with disease pathology, specifically round inclusions. These findings are the first to demonstrate unequivocally that abnormalities of peripherin splicing occur in ALS and that these splice variants may be intrinsically involved in the generation of disease pathology.

It is interesting to speculate how peripherin splicing abnormalities could occur in ALS. In this regard, the nuclear DNA/ RNA binding protein TAR DNA binding protein-43 (TDP-43) has been identified previously as a component of ubiquitinated inclusions in ALS (Arai et al., 2006; Neumann et al., 2006), and we have shown that peripherin and TDP-43 are colocalized to the same inclusions (Sanelli et al., 2007). One of the known functions of TDP-43 is to act as a splicing factor (Buratti and Baralle, 2001), and it is therefore tempting to speculate that the deregulated splicing we have observed for peripherin may be causally linked to abnormalities of TDP-43. This will be the subject of future investigations.

\section{References}

Arai T, Hasegawa M, Akiyama H, Ikeda K, Nonaka T, Mori H, Mann D, Tsuchiya K, Yoshida M, Hashizume Y, Oda T (2006) TDP-43 is a component of ubiquitin-positive tau-negative inclusions in frontotemporal lobar degeneration and amyotrophic lateral sclerosis. Biochem Biophys Res Commun 351:602-611.
Beaulieu JM, Nguyen MD, Julien JP (1999a) Late onset of motor neurons in mice overexpressing wild-type peripherin. J Cell Biol 147:531-544.

Beaulieu JM, Robertson J, Julien JP (1999b) Interactions between peripherin and neurofilaments in cultured cells: disruption of peripherin assembly by the NF-M and NF-H subunits. Biochem Cell Biol 77:41-45.

Buratti E, Baralle FE (2001) Characterization and functional implications of the RNA binding properties of nuclear factor TDP-43, a novel splicing regulator of CFTR exon 9. J Biol Chem 276:36337-36343.

Clark F, Thanaraj TA (2002) Categorization and characterization of transcript-confirmed constitutively and alternatively spliced introns and exons from human. Hum Mol Genet 11:451-464.

Clement AM, Nguyen MD, Roberts EA, Garcia ML, Boillee S, Rule M, McMahon AP, Doucette W, Siwek D, Ferrante RJ, Brown Jr RH, Julien JP, Goldstein LS, Cleveland DW (2003) Wildtype nonneuronal cells extend survival of SOD1 mutant motor neurons in ALS mice. Science 302:113-117.

Corbo M, Hays AP (1992) Peripherin and neurofilament protein coexist in spinal spheroids of motor neuron disease. J Neuropathol Exp Neurol 51:531-537.

Durham HD, Roy J, Dong L, Figlewicz DA (1997) Aggregation of mutant $\mathrm{Cu} / \mathrm{Zn}$ superoxide dismutase proteins in a culture model of ALS. J Neuropathol Exp Neurol 56:523-530.

Hays AP (2006) Pathology of amyotrophic lateral sclerosis. In: Amyotrophic lateral sclerosis (Mitsumoto H, Przedborski S, Gordon PH, eds), pp 43-80. New York: Taylor and Francis Group.

Hays AP, Naini A, He CZ, Mitsumoto H, Rowland LP (2006) Sporadic amyotrophic lateral sclerosis and breast cancer: hyaline conglomerate inclusions lead to identification of SOD1 mutation. J Neurol Sci 242:67-69.

He CZ, Hays AP (2004) Expression of peripherin in ubiquinated inclusions of amyotrophic lateral sclerosis. J Neurol Sci 217:47-54.

Ince PG, Lowe J, Shaw PJ (1998a) Amyotrophic lateral sclerosis: current issues in classification, pathogenesis and molecular pathology. Neuropathol Appl Neurobiol 24:104-117.

Ince PG, Tomkins J, Slade JY, Thatcher NM, Shaw PJ (1998b) Amyotrophic lateral sclerosis associated with genetic abnormalities in the gene encoding $\mathrm{Cu} / \mathrm{Zn}$ superoxide dismutase: molecular pathology of five new cases, and comparison with previous reports and 73 sporadic cases of ALS. J Neuropathol Exp Neurol 57:895-904.

Kan Z, States D, Gish W (2002) Selecting for functional alternative splices in ESTs. Genome Res 12:1837-1845.

Landon F, Lemonnier M, Benarous R, Huc C, Fiszman M, Gros F, Portier MM (1989) Multiple mRNAs encode peripherin, a neuronal intermediate filament protein. EMBO J 8:1719-1726.

Landon F, Wolff A, de Nechaud B (2000) Mouse peripherin isoforms. Biol Cell 92:397-407.

Lariviere RC, Beaulieu JM, Nguyen MD, Julien JP (2003) Peripherin is not a contributing factor to motor neuron disease in a mouse model of amyotrophic lateral sclerosis caused by mutant superoxide dismutase. Neurobiol Dis 13:158-166.

McLean J, Xiao S, Miyazaki K, Robertson J (2008) A novel peripherin isoform generated by alternative translation is required for normal filament network formation. J Neurochem, in press.

Migheli A, Pezzulo T, Attanasio A, Schiffer D (1993) Peripherin immunoreactive structures in amyotrophic lateral sclerosis. Lab Invest 68:185-191.

Neumann M, Sampathu DM, Kwong LK, Truax AC, Micsenyi MC, Chou TT, Bruce J, Schuck T, Grossman M, Clark CM, McCluskey LF, Miller BL, 
Masliah E, Mackenzie IR, Feldman H, Feiden W, Kretzschmar HA, Trojanowski JQ, Lee VM (2006) Ubiquitinated TDP-43 in frontotemporal lobar degeneration and amyotrophic lateral sclerosis. Science 314:130-133.

Robertson J, Beaulieu JM, Doroudchi MM, Durham HD, Julien JP, Mushynski WE (2001) Apoptotic death of neurons exhibiting peripherin aggregates is mediated by the proinflammatory cytokine tumor necrosis factoralpha. J Cell Biol 155:217-226.

Robertson J, Kriz J, Nguyen MD, Julien JP (2002) Pathways to motor neuron degeneration in transgenic mouse models. Biochimie 84:1151-1160.

Robertson J, Doroudchi MM, Nguyen MD, Durham HD, Strong MJ, Shaw G, Julien JP, Mushynski WE (2003) A neurotoxic peripherin splice variant in a mouse model of ALS. J Cell Biol 160:939-949.

Rosen DR, Siddique T, Patterson D, Figlewicz DA, Sapp P, Hentati A, Donaldson D, Goto J, O’Regan JP, Deng HX, Rahmani Z, Krizus A,
McKenna-Yasek D, Cayabyab A, Gaston SM, Berger R, Tanzi RE, Halperin JJ, Herzfeldt B, Van Den Bergh R, et al. (1993) Mutations in Cu/Zn superoxide dismutase gene are associated with familial amyotrophic lateral sclerosis. Nature 362:59-62.

Sanelli T, Xiao S, Horne P, Bilbao J, Zinman LH, Robertson J (2007) Evidence that TDP-43 is not the major ubiquitinated target in the pathological inclusions of ALS. J Neuropathol Exp Neurol 66:1147-1153.

Sarria AJ, Nordeen SK, Evans RM (1990) Regulated expression of vimentin cDNA in cells in the presence and absence of a preexisting vimentin filament network. J Cell Biol 111:553-565.

Wong J, Oblinger MM (1990) Differential regulation of peripherin and neurofilament gene expression in regenerating rat DRG neurons. J Neurosci Res 27:332-341.

Xiao S, McLean J, Robertson J (2006) Neuronal intermediate filaments and ALS: a new look at an old question. Biochim Biophys Acta 1762:10011012 . 